\title{
Transverse damage simulation and analysis of carbon nanotube reinforced composite
}

\author{
Dan $\mathrm{Hu}^{1, \mathrm{a}}$, Chenghong Duan ${ }^{1, \mathrm{~b}}$, Yuan Sha ${ }^{1, \mathrm{c}}$ \\ ${ }^{1}$ CAE, Beijing University of Chemical Technology, Beijing, 100029, China \\ ahudannn@163.com, 'duanchenghong@163.com, 'shayuan6216@yeah.net
}

Keywords: CNT, cohesive element, damage, random distribution model

\begin{abstract}
The carbon nanotube (CNT) composite material model containing interfaces was constructed in this paper. With the aid of the ABAQUS's cohesive element and user-defined field (USDFLD) technologies, the damage beginning and evolution of the composite model under transverse load was simulated in the way of material degradation. The effect of the random distribution of CNTs in the matrix was considered. Respectively, the maximum tensile stress and Von Mises strength theory was applied as failure criterion in this analysis, and their results were compared.
\end{abstract}

\section{Introduction}

Since Ajayan first made the CNT composite by adding CNTs to the polymer matrix in 1994, the CNTs have been widely used in all kinds of materials for its excellent mechanical performance [1]. Usually adding a small quantity of nanophase can greatly improve the whole mechanical properties of the composite, but it will also bring some side effects, such as interface flaw, CNTs aggregation [2, 3], etc. These problems have limited the developments and application of CNT composite materials on a certain degree.

The interface links the CNT and the matrix, its property plays a very important role in the macroscopic behavior of the composites materials. Terumasa Tsuda [4] measured the shearing strength of multi-walled CNT-PEEK interface, 3.5 7MPa, by pulling out test, and if it had been treated in the condition of $1 \mathrm{MPa}$ pressure and 573K temperature for 1 hour, the shearing strength of the interface could be improved to 6 14MPa. Surface modification has been frequently used in improving the composite property, Florian H. Gojny [5] strengthened the tie between the CNT and the epoxy matrix by modifying the multi-walled CNT surface. As to the distribution of the CNTs, Xiaolong Jia [6] added $\mathrm{Fe}_{3} \mathrm{O}_{4}$ particles to the CNTs, and made the CNTs distribute more orderly in the deionized water with the help of magnetic field, in this way the strength and modulus of the carbon nanotube-epoxy composite improved greatly. Longbin Qiu [7] also obtained well-aligned CNTs composite by synthesizing the nanotubes array with the CVD method, this made the material strength a magnitude order higher.

The efforts of scholars around the world have made the CNT composite developed rapidly and more widely applied. On this basis, CNT composite model containing interface was established in this paper, with the ABAQUS's cohesive element and user-defined field technologies, the composite damage process under transverse load was studied. Also the effect of CNTs' random distribution was considered.

\section{Model establishment}

Python script was used to realize Parametric modeling in this paper. The representative volume model containing matrix, interfaces and 9 CNTs was constructed as shown in Fig.1. The volume fraction of the CNTs is $5 \%$, the axial thickness of the model is $2.65 \mathrm{~nm}$, and the other dimensions are listed in Table.1. The CNTs and matrix were both meshed with element C3D8R, while the interface was meshed with the cohesive element COH3D8. The finite element model is shown in Fig.2. 


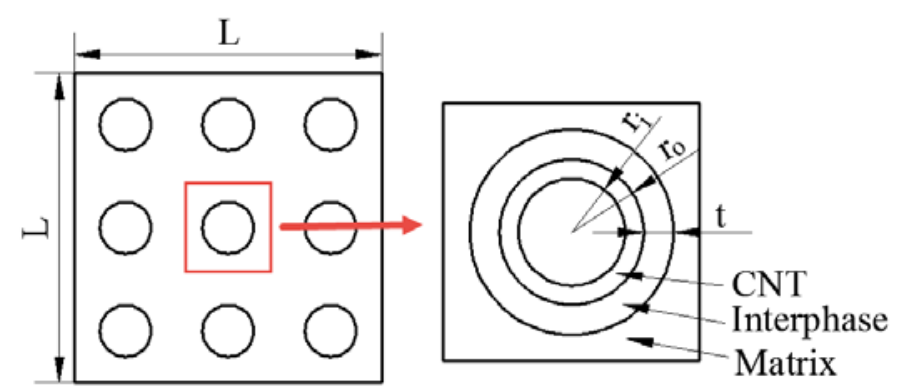

Fig. 1 Geometry model

Table 1 Dimensions of the model

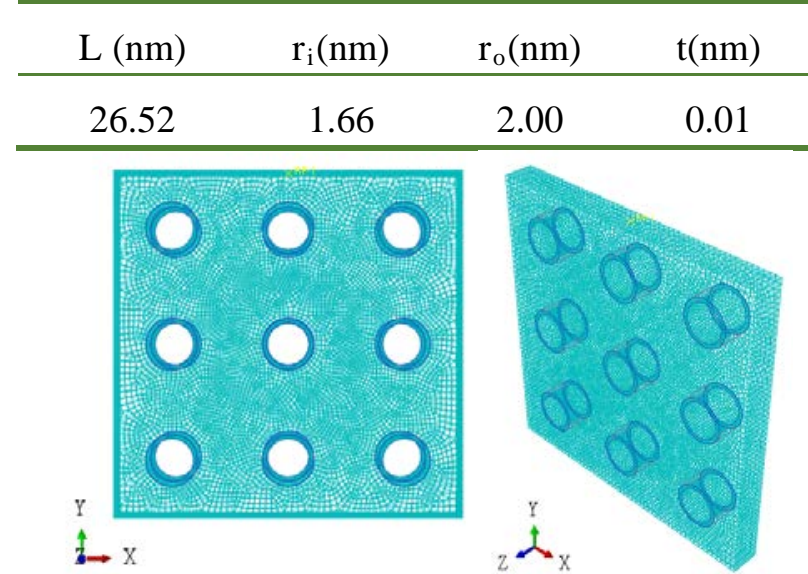

Fig. 2 Finite element model

As for boundary conditions, upward displacement load was applied on the upper surface of the model, to the other five surfaces, only the normal displacements were constraint.

CNTs, matrix and interface are all isotropic material in this paper, material property parameters are listed in Table.2.

Table 2 Material property parameters

\begin{tabular}{cccc}
\hline Material properties & CNT & Matrix & Interface \\
\hline $\begin{array}{c}\text { Young's } \\
\text { modulus(GPa) }\end{array}$ & 1000 & 3.5 & 5 \\
\hline Poisson's ratio & 0.34 & 0.48 & 0.48 \\
\hline Strength(MPa) & $\infty$ & 100 & 50 \\
\hline
\end{tabular}

\section{Solving method}

The cohesive element provided by ABAQUS can simulate the interface behavior between two different materials conveniently. In this paper bilinear load-displacement cohesive mode is applied to simulate the damage process of the interface between the CNT and matrix. The curve equation can be expressed as:

$$
\sigma= \begin{cases}\frac{\sigma_{0}}{\delta_{0}} \delta & \left(\delta \leq \delta_{0}\right) \\ \sigma_{0} \frac{\delta_{f}-\delta}{\delta_{f}-\delta_{0}} & \left(\delta_{f} \geq \delta>\delta_{0}\right) \\ 0 & \left(\delta \geq \delta_{f}\right)\end{cases}
$$

Where $\sigma_{0}$ is the initial failure stress, $\delta_{0}$ is the initial failure displacement, $\delta_{\mathrm{f}}$ is the complete failure displacement. When: $\delta<\delta_{0}$, the material is in the elastic state; $\delta=\delta_{0}$, the damage begins and the stress 
reaches the critical value $\sigma_{0}$, this is the initial failure stage; $\delta \geq \delta_{\mathrm{f}}$, the material completely damage, and loses the carrying capacity. In the damage evolution stage the stiffness weakening coefficient $\mathrm{D}$ is introduced. $\mathrm{D}=0$, that means no damage or the beginning of the damage, and $\mathrm{D}=1$ means complete damage. The cohesive model's constitutive relation in the damage evolution stage can be expressed as:

$$
\sigma=\left[\begin{array}{l}
\sigma_{n} \\
\sigma_{s} \\
\sigma_{t}
\end{array}\right]=\left[\begin{array}{lll}
(1-D) K_{n n} & & \\
& (1-D) K_{s s} & \\
& & (1-D) K_{t t}
\end{array}\right]\left[\begin{array}{l}
\varepsilon_{n} \\
\varepsilon_{s} \\
\varepsilon_{t}
\end{array}\right]
$$

Where, $\sigma_{\mathrm{n}}, \sigma_{\mathrm{s}}, \sigma_{\mathrm{t}}$ are normal stress, first shear direction stress and second shear direction stress, $\varepsilon_{\mathrm{n}}, \varepsilon_{\mathrm{s}}$, $\varepsilon_{\mathrm{n}}$, are the corresponding strain, and $\mathrm{K}_{\mathrm{nn}}, \mathrm{K}_{\mathrm{ss}}, \mathrm{K}_{\mathrm{tt}}$ are the corresponding modulus.

For the matrix, ABAQUS's USDFLD was used to simulate its damage process. As the load increases, limited degradation will be done to the matrix if stress, strain or other parameters reach the critical value of the considered criterion. Both the maximum tensile stress strength theory, Eqs.3 (usually for brittle material) and the Von Mises strength theory, Eqs.4 (usually for plastic material) are applied here.

$$
\begin{array}{ll}
\text { Damage: } & \sigma_{1} \geq[\sigma] \\
\text { No damage: } & \sigma_{1}<[\sigma] \\
\text { Damage: } & \sqrt{\frac{1}{2}\left(\left(\sigma_{1}-\sigma_{2}\right)^{2}+\left(\sigma_{2}-\sigma_{3}\right)^{2}+\left(\sigma_{3}-\sigma_{1}\right)^{2}\right)} \geq[\sigma] \\
\text { No damage: } & \sqrt{\frac{1}{2}\left(\left(\sigma_{1}-\sigma_{2}\right)^{2}+\left(\sigma_{2}-\sigma_{3}\right)^{2}+\left(\sigma_{3}-\sigma_{1}\right)^{2}\right)}<[\sigma]
\end{array}
$$

When the failure criterion is met, the modulus of the matrix will be reduced as Eqs.5:

$$
E_{d m}=d E_{m}
$$

Where $\mathrm{d}$ is the reduction factor. It was found that the trend of the stress-strain curve tended to be uniform as $d$ reduced. Taking into account the computational cost here, took 0.001 for $d$.

In order to observe the damage process of the matrix visually, state variable SDV1 was compiled by USDFLD. The state variable SDV1 is the ratio of equivalent stress to the matrix strength. When the equivalent stress becomes bigger than the matrix strength, the modulus of the matrix reduces, and the value of SDV1 is no longer changed when it reaches 1.

$$
S D V 1= \begin{cases}\frac{\sigma_{e}}{[\sigma]} & \left(\sigma_{e}<[\sigma]\right) \\ 1 & \left(\sigma_{e} \geq[\sigma]\right)\end{cases}
$$

The CNT's properties are excellent, and its strength is much higher than the matrix, so its modulus will not be reduced.

\section{Result analysis}

Fig. 3 shows the SDV1 contour of the model under the two different strength theories when the strain is $5 \%$.

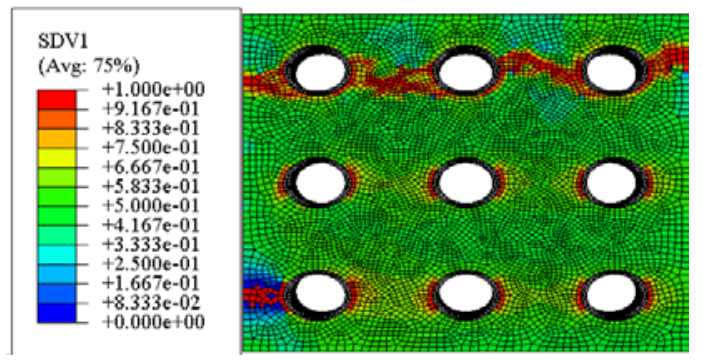

(a) Maximum tensile stress criterion

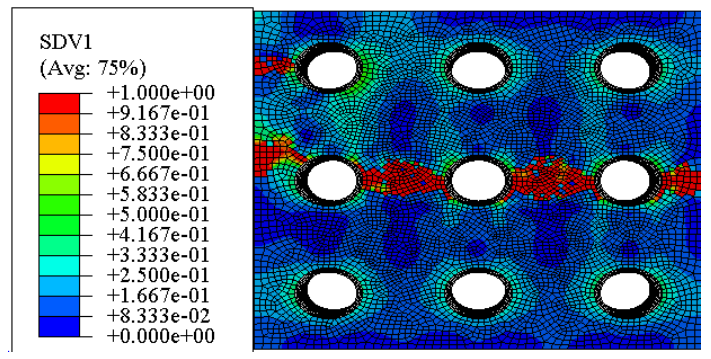

(b) Von Mises criterion

Fig.3 SDV1 contour of the model with 5\% strain

Analysis of the detailed damage process: 
1) Under both two strength criterion, damage began from the matrix elements next to the interface, and then extended to the adjacent CNT. Finally the damage region was like the Chinese character “一” penetrating the whole model, and vertical to the load direction.

2) Under the maximum tensile stress criterion, damage began when the strain reached $0.6 \%$, and the final failure region went through the first row of the CNTs. Under the Von Mises criterion, damage began when the strain reached $1.09 \%$, and the final failure region went through the second row of the CNTs.

3) In the undamaged region of the matrix, the average stress under the maximum tensile stress criterion was larger than that under the Von Mises criterion.

4) In the undamaged region of the matrix, the stress level was still high near the interface.

Under the maximum tensile stress criterion and Von Mises criterion, the damaged elements' location of the interfaces were different. Fig. 4 shows the weakening coefficient contour of the interface stiffness under the two different strength theories when the strain reaches 5\%.

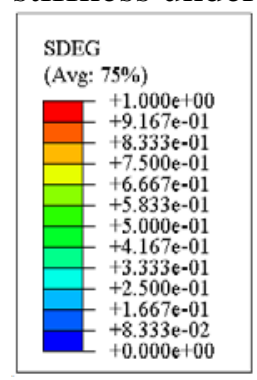

(a)Maximum tensile stress criterion
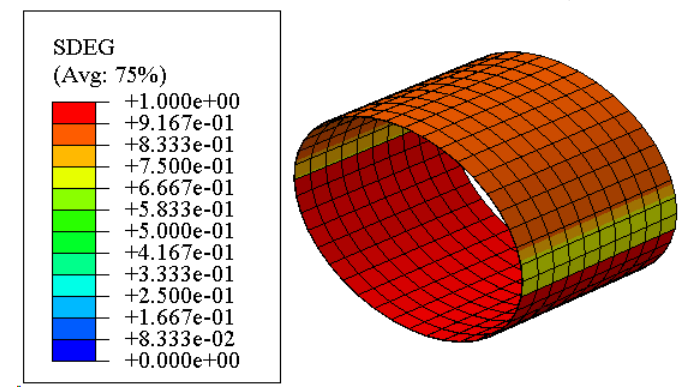

(b)Von Mises criterion

Fig.4 Stiffness weakening coefficient contour of the interface with $5 \%$ strain

Under both two criterion, interface damage began when the strain reached $0.33 \%$, while the damage evolution under the maximum tensile stress criterion was faster. Because the limited degradation was applied, the interface was damaged incompletely, but the stiffness weakening coefficient D (i.e. the SDEG value) reached quite a high level, so it could be considered that partial failure occurred to the interface. The locations of the damaged regions of the interface were different from that of the matrix, the interface's damaged regions were the upper and lower parts that vertical to the transverse load. The left and right sides of the interface were damaged slightly.

In order to analyze the effect of the CNTs distribution, five random models were established by using Python script to generate random numbers. See Fig.5.

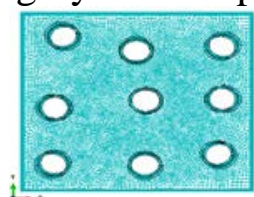

MODEL T1

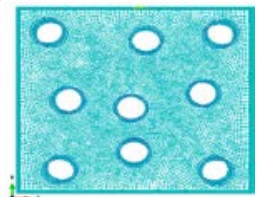

MODEL T2

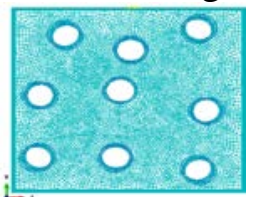

MODEL T3

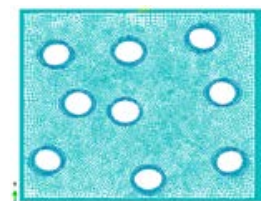

MODEL T4

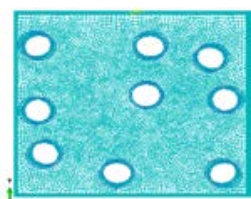

MODEL T5

Fig.5 Five random models

After analysis of different random models, it was found that damage always began between the two most closed CNTs, and then extended through the whole model transversely. As an example, Fig.6 shows the SDV1 contour of the MODEL T4 with 5\% strain.

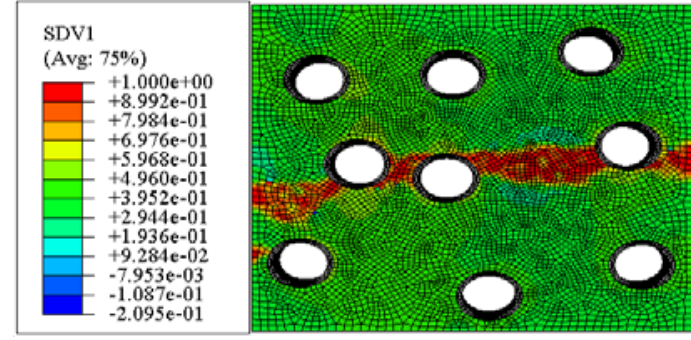

(a)Maximum tensile stress criterion

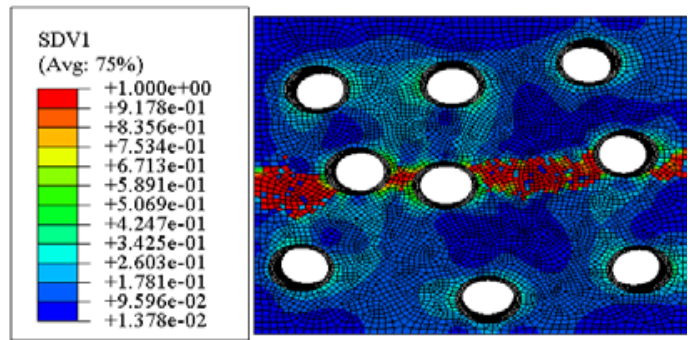

(b)Von Mises criterion

Fig.6 SDV1 contour of the MODEL T4 with 5\% strain

Analysis of the five random models' calculation results: 
1) Under the maximum tensile stress criterion, the maximum stress and corresponding strain of the five model are: $75.88 \mathrm{MPa}(0.57 \%), 75.75 \mathrm{MPa}(0.57 \%), 74.99 \mathrm{MPa}(0.55 \%), 73.41 \mathrm{MPa}$

$(0.54 \%), 72.88 \mathrm{MPa}(0.52 \%)$. While the CNTs-orderly-distributed model's maximum stress and corresponding strain is $76.76 \mathrm{MPa}(0.58 \%)$, so it can be seen that the strength of the random model has been weakened slightly.

2) Under the maximum tensile stress criterion, as for the stress-strain curves of different models, there is no obvious regularities after the maximum stress point, the stresses present a declining trend generally. After the strain reaching about $0.8 \%$, the curves turn into a relatively stable stage, and the stable values of different curves diverse.

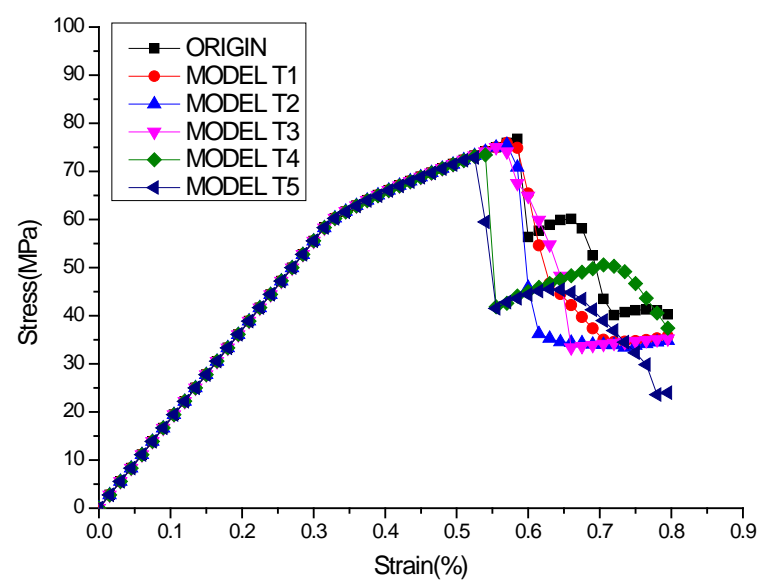

Fig.7 Stress-strain curves of the random models under the maximum tensile stress criterion

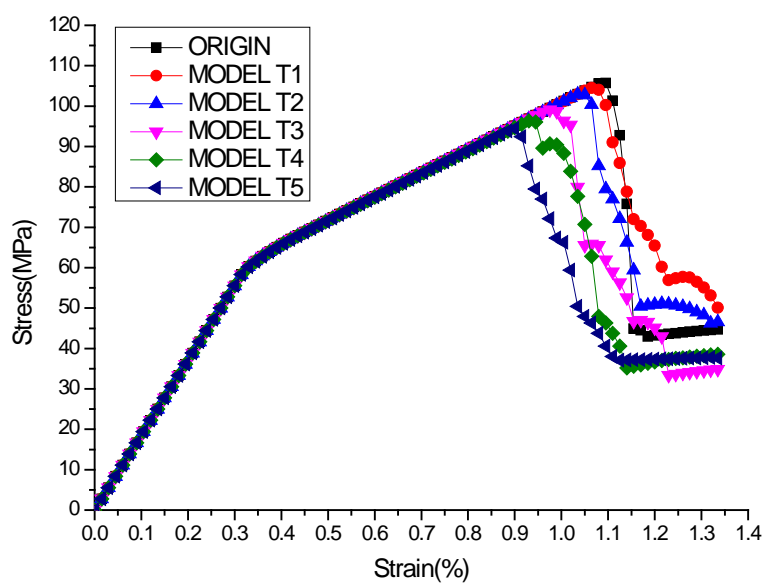

Fig.8 Stress-strain curves of the random models under the Von Mises criterion

3) Under the Von Mises criterion, the maximum stress and corresponding strain of the five models are: 104.48MPa (1.06\%), 102.93MPa (1.03\%), 99.11MPa (0.97\%), 96.10MPa (0.94\%), 94.44MPa $(0.88 \%)$. While the CNTs-orderly-distributed model's maximum stress and corresponding strain is $105.70 \mathrm{MPa}(1.09 \%)$, the strength of the random model has also been weakened.

4) Under the Von Mises criterion, the stress-strain curves' trends of different models tend to be the same after the maximum stress point. They all decline rapidly, then turn into a relatively stable stage after the strain reaching about $1.4 \%$, and the stable values diverse. 


\section{Conclusion}

In this paper cohesive element and USDFLD provide by ABAQUS were used to model the interface and matrix damage of the CNTs composite under the transverse load. Visually the damage process were presented.

Under the transverse load, damage first began at the interface zone that vertical to the load, then extended to the matrix. The final failure region appeared to be the Chinese character “一”, and was vertical to the load direction. The properties of the matrix and interface are the key factors to the composites transverse performance.

As to the CNTs-random-distributed models under the transverse load, damage usually began between the two most closed CNTs, and then extended to the adjacent CNTs until penetrating the whole model. The random distribution led to the transverse strength weakening on a certain degree.

\section{References}

[1]. Ajayan P M, Stepthan O, Colliex C, et al, Aligned carbon nanotube arrays formed by cutting a polymer resin-nanotube composite, J. Science. 265 (1994) 1212-1214.

[2]. Gkikas G, Barkoula NM, Paipetis AS, Effect of dispersion condition on the thermos-mechanical and toughness properties of multi-wall carbon nanotubes reinforced epoxy, J. Composites Part B. 6 (2012) 2697-2705.

[3]. Shaffer MSP, Fan X, Windle AH, Dispersion and packing of carbon nanotubes, J. Carbon. 11 (1998) 1603-1612.

[4]. Terumasa Tsuda, Toshio Ogasawara, Fei Deng, et al, Direct measurements of interfacial shear strength of multi-walled carbon nanotube/PEEK composite using a nano-pullout method, J. Composites Science and Technology. 71 (2011) 1295-1300.

[5]. Florian H. Gojny, Jacek Nastalczyk, Zbigniew Roslaniec, et al, Surface modified multi-walled carbon nanotubes in CNT/epoxy-composites, J. Chemical Physics Letters. 370 (2003) 820-824.

[6]. Xiaolong Jia, Wusheng Li ,Xianjuan Xu, et al, Numerical Characterization of Magnetically Aligned Multiwalled Carbon Nanotube-Fe3O4 Nanoparticle Complex, J. Applied Materials \& Interfaces. 7 (2015) 3170-3179.

[7]. Longbin Qiu, Xuemei Sun, Zhibin Yang, et al, Preparation and application of the Well-aligned carbon nanotube /new high polymer composite material, J. Acta Chimica Sinica. 70 (2012) 1523-1532. 